\title{
SORELLE DI QUERELLE. POETESSE DELL’ AL-ANDALUS, TROBAIRITZ E POETESSE ITALIANE DEL DUECENTO E DEL TRECENTO ${ }^{1}$
}

\author{
Daniele Cerrato ${ }^{2}$
}

\begin{abstract}
Sorelle di Querelle. Poetesse dell'al-Andalus, trobairitz e poetesse italiane del Duecento e Trecento

Riassunto: Il dibattito della Querelle des Femmes in Italia si sviluppa soprattutto durante il XV e XVI secolo attraverso vari trattati di autori ed autrici. In realtà l'inizio della Querelle des Femmes si potrebbe far concidere con le poetesse italiane del Duecento e del Trecento. L'articolo analizza come alcuni dei temi presenti nei testi di queste autrici si possono far risalire ad una tradizione di poesia femminile che comprende le poetesse dell'al-Andalus e le trobairitz. Parole chiave: Querelle des Femmes, poetesse italiane, poetesse al-Andalus, trobairitz, genealogia.
\end{abstract}

Sisters of Querelle. Al-Andalus Women Poets, Trobairitz and Italian Women Poets in the 13th and 14th Centuries

Abstract: The debate on the Querelle des Femmes during the $16^{\text {th }}$ and $17^{\text {th }}$ centuries in Italy is based on different male and female authors' dissertations. This Querelle des Femmes can be traced back to Italian women poets in the $13^{\text {th }}$ and $14^{\text {th }}$ centuries. This paper analyses some topics dealt with by these Italian women poets and studies the features they share with preceding al-Andalusian women poets and trobairitz.

Key words: Querelle des Femmes, Italian women poets, al-Andalusian women poets, trobairitz, genealogy.

In Italia, la Querelle des Femmes, il dibattito/polemica legato ai meriti e alla dignità delle donne, trova ampio spazio durante i secoli XV e XVI, in una serie di trattati composti da Mario Equicola, Galeazzo Capra, Torquato Tasso, ai quali si affiancano i testi di autrici quali Isotta Nogarola, Arcangela Tarabotti, Moderata Fonte, Cassandra Fedele, Lucrezia Marinella. Si tratta di un confronto letterario che si sviluppa soprattutto

\footnotetext{
${ }^{1}$ Data di ricezione: 02/12/2015.

Data di accettazione: 20/12/2015.

2“Contrato Puente Posdoctoral VPPI-US", Departamento de Filologias Integradas, Universidad de Sevilla; $\checkmark$ dcerrato@us.es. Il presente studio rientra all'interno del piano di attività e ricerche derivate dal "Contrato Puente Posdoctoral del VPPI-US".
} 
attraverso la prosa, ma vi prenderanno parte anche poetesse tra cui Laura Terracina, Laura Battiferri, Veronica Gambara e Veronica Franco ${ }^{3}$.

In realtà, come già sottolineato da Mercedes Arriaga (2008), l'inizio della Querelle in Italia si potrebbe anticipare di un paio di secoli e far risalire ai testi di poetesse italiane del Duecento e del Trecento quali Monna Nina, Compiuta Donzella, il gruppo di petrarchiste marchigiane (comprendenti Ortensia da Guglielmo, Giustina Levi Perotti, Livia del Chiavello, Leonora della Genga, Elisabetta Trebbiani) e le bolognesi Giovanna Bianchetti Bonsignori e Bartolomea Mattugliani ${ }^{4}$

Il presente articolo si propone di dimostrare come la produzione delle poetesse italiane del Duecento e del Trecento affondi le proprie radici in una tradizione anteriore, che si può far risalire alle poetesse dell'al-Andalus e alle trobairitz. Pur consapevoli del differente o culturale e sociale nel quale queste autrici vivono e scrivono, crediamo che le tre generazioni di poetesse possano essere accomunate, dal momento che i loro testi sviluppano alcuni dei temi che caratterizzeranno la Querelle de femmes come l'intraprendenza amorosa femminile, la difesa del ruolo della donna nella cultura, il confronto con gli uomini sul terreno letterario 5 .

Per tutte, la scrittura costituisce uno strumento decisivo per una presa di coscienza della condizione femminile e una rivendicazione dei propri diritti, che però non si manifesta solo attraverso le composizioni poetiche, ma anche attraverso la condivisione di spazi di creazione con i colleghi uomini. La corte e la città si presentano come luoghi di convivenza ideali per facilitare lo scambio poetico e l'affermazione di testi in lingua volgare.

${ }^{3}$ Per un approfondimento riguardo alla Querelle des femmes in Italia si rimanda a Ramirez Almazán, Martín Clavijo, Aguilar González, Cerrato 2011, Cerrato 2013, Gonzalez de Sande \& Gonzalez de Sande 2013, Gonzalez de Sande 2013.

${ }^{4}$ Sul contributo delle poetesse del Duecento e Trecento all'interno della Querelle des Femmes in Italia si veda Arriaga Flórez, Cerrato, Rosal Nadales 2012.

${ }^{5}$ Rosamaría Aguadé Benet nel suo articolo "Cristine de Pizan y las trobairitz" sottolineava il legame tra Christine De Pizan e le stesse trobairitz dal momento che l'autrice de la Cité des dames nei suoi testi riprendeva e ampliava il discorso riguardante la posizione e il ruolo delle donne nel mondo aristocratico dei secoli XII e XIII che quest'ultime avevano già trattato nei loro componimenti. 
Sebbene il contesto culturale in cui le poetesse italiane del Duecento e Trecento compongono i loro testi, non sia mai stato oggetto di studi approfonditi e manchino documenti e testimonianze in grado di permettere una ricostruzione esaustiva, è possibile ipotizzare che abbiano potuto leggere e consultare alcuni dei poemi provenienti dalle tradizioni precedenti. In ogni caso, queste autrici sembrano essere certamente a conoscenza di molte strutture e topoi della tradizione provenzale e arabo-andalusa che trasferiscono e reinterpretano nei loro componimenti. Un altro elemento che, fin dalle poetesse dell'al-Andalus ${ }^{6}$, contraddistingue questi gruppi di autrici, è il fatto che le informazioni che le riguardano ci siano giunte per citazioni di poeti a loro contemporanei, o con i quali entrarono in relazione. Lo stesso dicasi per molti dei loro poemi che vengono tramandati perché costituiscono la risposta a poesie maschili, all'interno di scambi poetici o semplicemente attraverso citazioni.

Anche presso le trobairitz e le poetesse italiane dei primi secoli, troviamo autrici che vengono ricordate per corrispondenze con altri poeti, si pensi ad esempio a Monna Nina e Dante Da Maiano, o citate da altri scrittori come nel caso di Compiuta Donzella, ricordata da Mastro Torrigiano e Guittone d'Arezzo ${ }^{7}$.

Nella tradizione dell'al-Andalus, vari testi con voce femminile, inoltre, si presentano in forma anonima attraverso il genere poetico della "moaxaja", che si conclude con una "jarcha", glossa che rimanda ad un ambito popolare ricollegabile al genere dei frauenlieder ${ }^{8}$.

Questo genere letterario sembra trovare affinità con molti dei brevi testi che, come nel caso del sonetto anonimo "Pàrtite amore adeo", erano contenuti nei Memoriali

\footnotetext{
${ }^{6}$ Con poetesse dell'al-Andalus ci si riferisce al gruppo di autrici che composero versi durante il periodo in cui la penisola Iberica era sotto la giurisdizione araba, dal secolo VIII fino al XV.

${ }^{7} \mathrm{Si}$ vedano ad esempio le composizioni di Mastro Torrigiano "Esser donzella di trovare dotta" e "S'una donzella di trovar s'ingegna" e la lettera V contenuta nell'epistolario di Guittone.

${ }^{8} \mathrm{Si}$ tratta di uno schema, che, come osserva Elvira Gangutia in "Poesía griega de amigo y poesía arábigoespañola" (Emerita 40, 1972. 329-396), si può far risalire già alla poesia greca arcaica e al culto mesopotamico di Inanna/Istar/Astarté. Per quanto riguarda la tradizione dei frauenlieder si veda Muñoz Saavedra, Búa Carballo 2007 e Millet 2006.

${ }^{9}$ Il sonetto che Angelo Gianni attribuisce a Nina Siciliana descrive la separazione di due amanti al sopraggiungere dell'alba ed è scandito da una voce di donna e anche il personaggio maschile viene descritto e determinato dalle parole dell'amata come avviene nel sonetto "Tapina me che amava uno sparviero" della stessa autrice. Cfr. Gianni 1997. Anch'esse quasi simili a Dio. Le donne nella storia della letteratura
} 
dell'Archivio notarile di Bologna, dove a partire dal 1265 si iniziarono a trascrivere i vari atti del Comune e, tra gli spazi bianchi, i notari bolognesi inserirono molti componimenti in lingua volgare.

Tra i testi anonimi della tradizione dell'al-Andalus, si trovano le cosiddette canzoni di richiesta d'amore, in cui la donna si rivolge direttamente all'amato e in cui il desiderio femminile viene espresso liberamente, senza nessun tipo di finzione retorica, limitazione o censura. Anche in questo caso abbiamo esempi nella tradizione italiana, basti ricordare il testo anonimo "Oi bona gente oditi ed intenditi" dove due cognate si lamentano delle poche attenzioni dei mariti nei loro confroni, ma una tranquillizza l'altra dicendole di non preoccuparsi perchè ha invitato a pranzo un giovane che potrà soddisfare i loro desideri ${ }^{10}$.

Un altro tipo di componimento è quello della canzone di protesta, dove la donna si lamenta del trattamento ricevuto dall'amante. Questo genere troverà ampio sviluppo nella lirica trovadorica e anche nella tradizione italiana, all'interno della tenzone, come avviene nel caso del sonetto di Monna Nina "Qual sète voi, che cara profferenza"11, che appartiene alla scambio poetico con Dante da Maiano, e sembra testimoniare il raggiungimento di

italiana, in gran parte ignote o misconosciute dalle Origini alla fine dell'Ottocento, Lucca, Mario Barone: 14. /Partite, amore, adeo;/ché tropo çe se'stato./ Lo maytino è sonato,/ çorno me par che sia/ Partite, amore, adeo;/ che non fossi trovato/ in sí fina celata/como nui semo stati./ Or me bassa, oclo meo;/ tosto sia l'andata,/ tenendo la tornata/ como d'inamorati,/ sí che per spesso usato/ nostra çoglia renovi:/ nostro stato non trovi/ la mala çelosia./Pàrtite, amore, adeo/ e vane tostamente;/ ch'one toa cosa t'aço/ pareclata in presente./ "Il sonetto Tapina me che amava uno sparviero" si trova citato in Trucchi, Francesco. 1846. Poesie italiane inedite di dugento autori. Dall'origine della lingua infino al secolo decimosettimo, raccolte ed illustrate, Ranieri Guasti, Prato, II, vol.: 53. /Tapina me che amava uno sparviero;/ Amaval tanto ch'io me morìa; a lo richiamo ben m'era maniero,/ ed unque troppo pascer nol dovìa./ Or è montato e salito sì altero,/ assai più altero che far non solìa;/ ed è assiso dentro a un verziero,/ e un'altra donna l'averà in balìa./ Isparvier mio, ch'io t'avea nodrito;/ sonaglio d'oro ti facea portare,/ perché nell'uccellar fossi più ardito;/ or sei salito siccome lo mare,/ ed hai rotti li geti e sei fuggito/ quando eri fermo nel tuo uccellare./

10 "Oi bona gente, oditi et intenditi/ la vita che fa questa mia cognata./ La vita ch'ela fa vui l'odirite, /e se ve place voilave contare. / A lato se ne te sette gallette/ pur del meglior per poter ben conçare,/ e tutora dice che mor de sete/ensin ch'a lato non se 'l po a acostare:/né vin ne agua no la po saçare/ se'ella non pon la boch'a la stagnata/ [...] Cognata mia ched eo t'ò ditto,/ eo saço ben ch'ed ell'è mal a dire. Ma menarot'a casa un fanteletto/ e lui daremo ben mançar e bere/ e tu recherai del to vin bruschetto/e recheró del meo plen un barile./Quando gl'avremo dà mançar e bere,/çaschuna faça la soa cavalcata/".

${ }^{11}$ Qual sète voi, che cara profferenza'Qual sète voi, che cara profferenza/sì fate a me, senza pur voi mostrare?/Molto m'agenzeria vostra parvenza,/perchè ' 1 meo cor potessi dichiarare./Vostro mandato aggrada a mia intenza,/ in gioia mi conteìa d'udir nomare/lo vostro nome che fa profferenza/d'essere sottoposto a me innorare./Lo core meo pensar non si savrìa/Alcuna cosa che sturbasse amanza,/Così affermo e voglio ognor che sia./L'udire a voi parlare è voglia mia,/Se vostra penna ha buona consonanza/Col vostro core, od è tra lor resìa./ 
quell'equilibrio e quella reciprocità poetica presente nei testi delle poetesse del alAndalus e delle trobairitz. In questo testo Monna Nina si posiziona sullo stesso livello del suo interlocutore, già a partire dalla domanda diretta che gli rivolge in apertura di sonetto (“Qual sete voi, che cara profferenza / sí fate a me, senza pur voi mostrare?”). La sicurezza della poetessa sempre crescere con l'avanzare dei versi e oltre all'intraprendenza amorosa ("Molto m'agenzeria vostra parvenza") aumentano anche la decisione e l'autorevolezza di Nina ("Così affermo e voglio ognor che sia"). L'ultima terzina del sonetto ("L'udire a voi parlare è voglia mia/ se vostra penna ha buona consonanza/ col vostro cuore od è tra lor resia") che ironizza sul fatto che l'amore proclamato da Dante faccia parte solo di una finzione poetica, permette a Nina di incalzare il suo interlocutore e al tempo stesso distanziarsene, per poterlo osservare e giudicare.

Altro tema comune è quello dell'assenza dell'amante che ricorre in molte liriche anonime dell'al-Andalus, ed è presente anche in alcune liriche italiane, come ad esempio "Il lamento della sposa padovana"12.

Tra le varie autrici dell'al-Andalus si distinse, ad esempio, A'isa Al-Qurtubiyya che visse intorno al secolo $\mathrm{X}$, ed apparteneva ad una nobile famiglia cordovese. I suoi biografi raccontano che non vi fosse nessuna donna tra le nobili dell'al-Andalus che potesse competere con lei "per quanto riguarda la capacità di comprensione in doti poetiche e retoriche, in virtù, eloquenza e buon giudizio" (Garulo 1986: 57). Tra i suoi poemi si è conservata la sua risposta orgogliosa ad un poeta che la chiedeva in matrimonio:

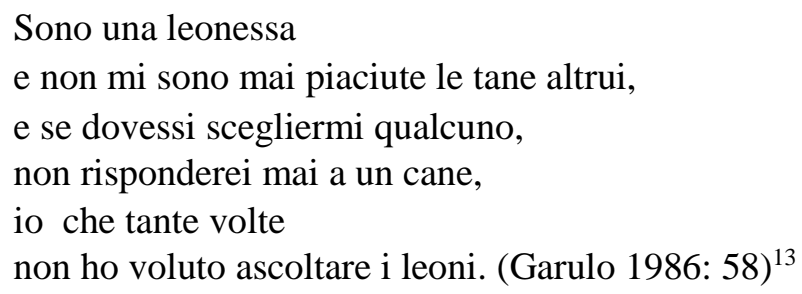

\footnotetext{
${ }^{12}$ A proposito di questo componimento, si veda ad esempio Massagrande, Matteo. 1995. Lamento della sposa padovana: quattro testi poetici e quattro incisioni, Belluno, Proposte d'arte Colophon.

${ }^{13}$ Tutte le traduzioni in italiano sono personali.
} 
Il richiamo alla natura e al mondo animale, che tradizionalmente viene accostato alle donne in maniera spregiativa e degradante, viene in questo caso completamente stravolto. A'isa se ne serve per autodeterminarsi e rinforzare il suo messaggio. La leonessa che rifiuta i "corteggiatori cani" diventa, quindi, un'efficace metafora della donna autorevole e che si distingue per la sua fierezza. La stessa struttura e lo stesso ribaltamento di prospettiva si riscontrà nella lirica del XIII secolo di Monna Nina "Tapina me che amava uno sparviero" 14 , dove si offre un'immagine non convenzionale della relazione uomo/donna e l'autrice raffigura l'amante come un rapace che ha saputo addomesticare.

Sempre nel secolo X, ad Almeria, visse Al-Gassaniyya. Si sono conservati solo sei versi di un suo panegirico amoroso, dove ricorda i momenti lieti trascorsi abbracciata al suo amante.

Ti rattristi che dicano: partiranno tutte le belle?

Come potrai resistere se andranno via da te?

La sua partenza è la morte, e se hai dubbi,

Vivi, raccoglierai le tristezze del loro allontanameno.

Con loro mi incontravo e alla loro ombra vivere era delizia, e il giardino della vita, radiante e profumato.

Notti felici nelle quali non temevo i rimproveri quando amavo, né mi spaventava l'abbandono quando eravamo insieme, quando il piacere ci assaltava e i desideri abbracciavamo come i rami che si abbracciano spinti dal vento.

Ah magari sapessi, ora che arriva la separazione, se mi amerai, dopo che siamo separati, come prima. (Garulo 1986: 67)

La stessa immagine dell'abbraccio e del giacere con l'amato è presente anche nei versi de "Estat ai en greu cossirier" della Contessa de Dia e "Tapina me che amava uno

\footnotetext{
${ }^{14}$ Lo stesso argomento si trova sviluppato anche nella lirica germanica, conosciuta come la "Canzone del falcone" del poeta del secolo XII Der Von Kuremberger. Si tratta di una composizione scritta in voce femminile che rappresenta certamente un modello di riferimento per Monna Nina quando compone il suo sonetto "Allevai un falcone per un anno/ormai domestico come io volevo/adorno di piume di oro,/volò fuggendo il falcone in altro cielo/Vidi allora volare il bel falcone:/lacci di seta brillavano alle zampe,/tutte le piume eran rosse e d'oro:/unisca Dio chi si vuole amare!/
} 
sparviero" di Monna Nina. Si tratta di immagini che rompono con lo stereotipo di passività femminile e raffigurano donne che si dimostrano risolute ed intraprendenti.

La figura che meglio incarna questo ideale di libertà ed indipendenza è certamente Wallada Bint Al Mustakfi o Wallada Almostacfi, conosciuta semplicemente con il nome di Wallada, la poetessa più nota del gruppo delle autrici dell'al-Andalus che visse a Cordoba tra il finire del secolo X e gli inizi del secolo XI, ricoprendo un ruolo di primo piano nella cultura e letteratura della città andalusa. Intorno al suo personaggio si sono costruiti vari aneddoti che hanno contribuito a creare il suo mito, come la leggenda che afferma che avesse fatto ricamare sul suo vestito alcuni versi che lei stessa aveva composto. Sul lato sinistro compariva "Allah mi ha creato per la gloria, e seguo orgogliosa il mio cammino", mentre sul lato destro "Offro al mio amante la mia guancia e do i miei baci a chi li desidera" (Rubiera Mata 1990: 103).

Della vasta produzione di Wallada, si conservano solamente nove poemi. Molti di questi sono riconducibili al suo legame con il poema Ibn Zaydun. I suoi versi scandiscono le varie fasi della relazione, tra passione, gelosie, tradimenti, fino al distacco e all'abbandono. Si trova ad esempio il topos dell'appuntamento notturno, che caratterizza anche altre liriche della produzione al-Andalus:

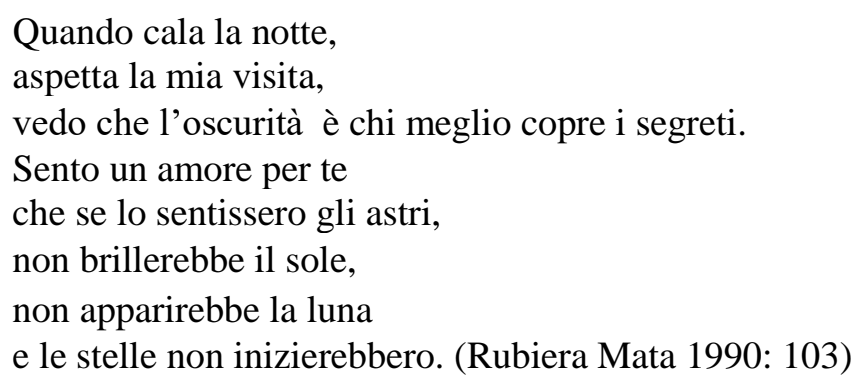

A questi versi di amore, fanno da contraltare le parole che Wallada fa giungere all'amante, in seguito ad un tradimento. La dolcezza e l'armonia lasciano spazio alle accuse e alle minacce: 
Se fossi stato giusto

per l'amore che c'è tra di noi,

non ameresti, né avresti preferito, una mia schiava.

Hai lasciato i rami dove fiorisce la bellezza

E hai scelto rami che non danno frutti.

Sai che sono la luna dei cieli,

ma hai scelto, per mia disgrazia, un pianeta oscuro. (Rubiera Mata 1990: 104)

La delusione di Wallada si trasforma in risentimento e rabbia, come ben racchiude la celebre satira dedicata sempre a Ibn Zaydun:

Il tuo soprannome è l'esagono, un epiteto che non ti abbandonerà neppure quando sarai morto: pederasta, puttaniere, adultero, bastardo, cornuto e ladro oscuro. (Rubiera Mata 1990: 104)

Un'altra poetessa molto nota fu certamente Hafsa Bint Al-Hayy Ar- Rakuniyya. Visse, probabilmente, durante la metà del XII secolo e apparteneneva ad una famiglia di Granada. Molti dei suoi versi conservatisi, fanno riferimento al tormentato amore con il poeta Abu Ya'far, conclusosi con la morte di quest'ultimo. Poemi in cui esprime il desiderio di poter incontrare l'amato, sono seguiti da componimenti dove lo rimprovera di interessarsi ad altre donne. Hafsa, in più occasioni, pone l'accento sulle mancanze e difetti dell'amante, esigendo un rapporto di reciprocità: "Smettila di enumerare le mie mancanze, ogni volta che ci vediamo, io non terrò conto delle tue, non tener conto tu delle mie" (Rubiera Mata 1990: 78).

In altri casi, i versi di amore brillano per la bellezza delle metafore, anche quando gli inviti sessuali si fanno più espliciti. Hafsa si dimostra, dunque, libera non solo nella sua maniera di vivere la vita, ma anche per come utilizza e interpreta il linguaggio in tutte le sue possibilità di espressione:

Vengo io da te o verrai tu a farmi visita? 
Il mio cuore sempre si curva verso i tuoi desideri.

Troverai rimedio alla sete e all'ardore del sole quando mi darai la benvenuta:

le mie labbra sono acqua dolce e fresca,

e i rami delle mie treccie offrono un' ombra generosa.

Rispondimi in fretta, non è bello, oh, mio Yamil,

far aspettare la tua Butayna ${ }^{15}$. (Rubiera Mata 1990: 83)

Anche Mut'a, schiava del celebre Ziryab, nel suo unico poema conservatosi, si rivolge nella stessa maniera all'amato:

Tu che nascondi la tua passione,

chi può nascondere il giorno?

Avevo un cuore, ma mi sono innamorata

ed è volato lontano da me,

era mio o in prestito? Amo un Qurasí

e per lui ho perso la timidezza. (Rubiera Mata 1990: 109)

L'amore non è però l'unico tema che caratterizza la produzione delle poetesse dell'al-Andalus e frequenti sono le rivendicazioni del proprio stato di donne e poetesse, come nel caso di Hafsa Al-Hiyariyya, vissuta nella stessa epoca e originaria di Guadalajara. Tra i suoi quattro poemi conservatisi, un chiaro esempio è rappresentato da questi versi:

Ho un amante a cui non piacciono i rimproveri

e quando lo lasciai, di orgoglio si riempì e mi disse:

"Hai visto qualcuno che mi assomigli?"

E anche io gli ho domandato:

"E tu hai incontrato chi mi faccia ombra?" (Garulo 1986: 67)

\footnotetext{
${ }^{15}$ Yamil era un celebre poeta arabo e Butayna la sua amata, si vuole dunque fare riferimento alla coppia di amanti perfetti per antonomasia.
} 
Infine, una poesia che insiste sull'importanza della cultura e della formazione femminile, è quella di Umm Al-Hasan Bint:

La bella lettera non serve alla scienza

è solamente un adorno nella carta;

lo studio è la mia meta e non desidero altra cosa,

poiché in base al suo sapere,

il giovane si eleva sui mortali. (Rubiera Mata 1990: 132)

Con i suoi versi Umm Al-Hasan Bint e, attraverso la metafora della bella calligrafia, prende le distanze dal ruolo di passività ed adorno attribuito alle donne e sottolinea l'importanza dello studio e della cultura come mezzo di emancipazione e potere. Si tratta di un tema che trova corrispondenza nei sonetti di Giustina Levi Perotti "Io vorrei pur drizzar queeste mie piume"16 e di Leonora Della Genga "Tacete o maschi a dir che la natura" ${ }^{17}$. Nel suo sonetto Giustina Levi Perotti introduce il tema della fama, associandolo alla donna, e rivendica uno spazio femminile nella cultura, affermando il diritto alla scrittura, ad essere poeta al pari degli uomini, rompendo con lo stereotipo che vuole la donna relegata e confinata all'ambito domestico (“all'ago e al fuso") e l'uomo destinato agli onori della vita pubblica (“al lauro o al mirto").

Sulla stessa linea si colloca Leonora Della Genga che in "Tacete o maschi a dir che la natura", non solo sfida gli uomini, ma afferma una superiorità femminile. Il suo sonetto riassume il nucleo della Querelle des femme intesa come dibattito filosofico che cerca di

\footnotetext{
${ }^{16}$ Io vorrei pur drizzar queste mie piume/ colà, Signor, dove il desìo m'invita,/ e dopo la morte rimanere in vita/ col chiaro di virtute inclito lume;/ ma il volgo inerte, che, dal rio costume vinto,/ ha d'ogni suo ben la via smarrita,/come degna di biasimo ognor m'addita,/ ch'ir tenti d'Elicona al sacro fiume./All'ago, al fuso, più ch'al lauro o al mirto/ (come se qui non sia la gloria mia),/ vuol ch'abbia sempre questa mente intesa./ Dimmi tu ormai che, per più dritta via/ a Parnaso te'n vai, nobile spirto,/dovrò dunque lasciar sì degna impresa?/

17 Tacete, o maschi, a dir che la Natura/ a far il maschio solamente intenda,/ e per formar la femmina non prenda/ se non contra sua voglia alcuna cura./ Qual invidia per tal, qual nube oscura/ fa che la mente vostra non comprenda/ com'ella in farle ogni sua forza spenda,/ onde la gloria lor la vostra oscura?/ Sanno le donne maneggiar le spade,/ sanno regger gl'imperi, e sanno ancora/trovar il cammin dritto in Elicona./ In ogni cosa il valor vostro cade,/ uomini, appresso a loro./ Uomo non fôra/ mai per tôrne di man pregio o corona./
} 
dimostrare che l'inferiorità femminile è una fallacia. Il testo di Leonora Della Genga inizia con l'invito degli uomini a tacere, invertendo la regola che da sempre imponeva alla donna il silenzio e contrasta l'arbitrarietà che a partire da Aristotele sosteneva una superiorità naturale dell'uomo.

Leonora Della Genga mette in evidenza le capacità femminili, attraverso elementi tradizionalmente associati agli uomini (la spada, la corona) e attraverso tutti gli ambiti da cui le donne vengono escluse, come la politica, la cultura e la guerra. Il testo anticipa inoltre il topico della eccellenza femminile che si troverà in autrici come Lucrezia Marinella, Moderata Fonte e Arcangela Tarabotti.

La relazione uomo-donna, nelle sue varie declinazioni è il tema centrale di molte delle composizioni delle poetesse trobairitz. Frequenti sono le tenzoni e i partimen, composizioni costruite sul dialogo tra due donne o una donna ed un uomo che disquisiscono su temi e questioni, spesso legate alle tematiche d'amore. Come sottolinea Rosamaría Aguadé Benet:

quella delle trobairitz era una voce distinta, che si esprimeva da un piano nel quale dominava l'osservazione dei loro sentimenti, l'esplorazione di certe emozioni che, al momento di cantarle, sembravano ancora più certe. E quasi potremmo dire che suggeriscono che la loro e quella dei trovatori è una poesia che si muoveva in mondi paralleli, nonostante formalmente il mondo fosse lo stesso, e che giocava con un vocabolario e con concetti simili. (Aguadé Benet 2011: 26)

Marirí Martinengo (1996) distingueva all'interno della produzione delle trobairitz due macrotematiche: quella legata all'amore declinato in tutte le sue fasi (innamoramento, lamento, perorazione, lite, etica amorosa) e quella sociale e politica che è dove si trovano maggiori spunti riconducibili alla Querelle. Nella maggiorparte dei casi si tratta di dialoghi e scambi poetici che si muovono nell'ambito dell'aristocrazia colta, come si può ricavare anche da alcuni riferimenti dei testi pervenutici e dalle vite delle trobairitz, dove spesso si dà risalto alla loro formazione letteraria. Il corpus di poesie di queste autrici non 
è molto vasto e presenta all'incirca venticinque testi, alcuni dei quali frammentati, mentre l'elenco di poetesse riconosciute e delle quali si hanno dati biografici è molto più ampio ${ }^{18}$.

Un altro aspetto che si ricollega alla Querelle des Femmes è il ruolo ricoperto da varie trobairitz nel favorire l'attività di altre poetesse, permettendo che questa tradizione poetica femminile potesse diffondersi e tramandarsi. Si tratta di un particolare che le accomuna anche al gruppo di petrarchiste marchigiane che lavorano a stretto contatto, appoggiandosi vicendevolmente ${ }^{19}$.

La prima figura nota è quella di Tibors de Sarenom, dal nome del castello di cui si pensa fosse signora. Visse presumibilmente tra la metà e la fine del XII secolo. C'è giunta una sua breve biografia in lingua occitana, dove si dice che fu cortese, raffinata, graziosa, molto colta, amata ed onorata dai suoi contemporanei. Costituì, insieme al suo primo marito Bertrand de Les Baux, un centro importante di cultura trovatoresca e si dice fosse divenuta la tutrice di Rimbaud D’Orange, che l'iniziò alla cultura cortese presso il suo centro. Le sue opere erano conosciute ancora nel secolo XVII, ma resta, solamente, un frammento di una sua canzone dal titolo "Bels dous amic, ben vos posc en ver dir":

Bel dolce amico, ben posso in verità che non mi mancò il desiderio un solo istante da quando voleste che vi avessi come amante neppure accade bello e dolce amico, che io non avessi voglia di vedervi spesso né ci fu stagione nella quale mi pentissi,

\footnotetext{
${ }^{18}$ Tra le autrici di cui si sono conservati testi e notizie biografiche si possono ricordare Tibors, La Contessa de Dia, Almucs de Castelnau, Iseut de Capio, Azalais de Porcairagues, Maria de Ventadorn, Alamanda, Garsenda, Isabel, Lombarda, Castelloza, Clara de Anduza, Azalais de Altier, Bieiris de Roman, Guillerma de Rosers, Germunda de Montpellier. A questo elenco vanno aggiunti i nomi di Alais, Iselda e Carenza, Dama H, la Genovese e Bona Domna, di cui si hanno testi, ma non notizie biografiche. Vi sono poi altri nomi che vengono citati da autori e studiosi come ad esempio Blanceman, domna N'Agnes, Caudairenga, Lisa di Londra, Guiscarda, Blankaflour, Huguette des Baulx, Marcabruna, Lauretta de Sade, Phanetta de Gantelmes, la moglie di Bertrand de Pezars, Margarida, Elionors, Sancha e Beatrice, la figlia di Ramon Berenguers. Inoltre, vanno tenuti presenti un buon numero di testi anonimi con voce femminile, che sono stati generalmente attribuiti a trovatori e invece, come sostengono vari studi, come ad esempio quelli di Angelica Rieger, potrebbero essere attribuiti a trobairitz.

${ }^{19}$ La trobairitz Tibors, ad esempio, accolse molte altre poetesse presso la corte di Le Baux, lo stesso fecero Ermengarda di Narbona a cui si rivolge Azalais de Porcairagues, e Maria di Ventadorm. Per quanto riguarda il contesto italiano va ricordato il ruolo di mecenate svolto da Livia del Chiavello che ospitò presso la sua corte e sostenne l'attività di Giovanna d'Arcangelo di Fiore.
} 
né mai capitò, che partiste irato.

che provassi gioia prima che foste tornato. (Martinengo 1996: 47)

Nel testo di Tibors, come in molti altri delle trobairitz, la voce femminile emerge con forza e la protagonista non si affida a stratagemmi retorici, nascondendosi dietro a finzioni poetiche, per esprimere i propri sentimenti. Si tratta di un passaggio fondamentale nel percorso di affermazione di un io femminile, perché la donna cessa di essere personaggio narrato, oggetto dedicatorio di poemi, icona vagheggiata ed assume il ruolo di creatrice e costruttrice di una nuova dimensione, dove con le parole riempie il silenzio a cui l'aveva destinata la letteratura maschile.

Tra le trobairitz, una delle più note è certamente la Contessa de Dia. Nella vita giunta fino a noi si dice che fu moglie di Guillermo di Poitiers e che poi si legò a Rimbaud di Orange, al quale dedicò molte belle canzoni ${ }^{20}$. Nelle poche note biografiche non si fa cenno al suo nome, ma, se si accetta la tradizione, si sarebbe chiamata Beatrice. Presso il centro di Les Baux, non lontano da Dia, probabilmente conobbe Rimbaud D'Orange. Delle numerose canzoni composte da Beatriz Contessa de Dia, un unicum è costituito da "A chantar m'er de so quieu non volria", la sola canzone di una trobadoritz comprensiva di notazione musicale.

La Contessa di Dia non canta l'amore coniugale/tradizionale, ma quello guidato dal desiderio e dalla passione. La massima espressione di questo sentimento si può ritrovare nella sua canzone forse più nota, "Estat ai en greu cossirier". In questo testo, l'autrice celebra l'amore più sensuale, che si distacca dalla ragione, per farsi corpo e carne:
Sono stata in grande affanno
per un cavaliere che ho avuto
come l'ho amato a dismisura
e voglio che sia sempre risaputo

\footnotetext{
${ }^{20}$ A distanza di oltre sei secoli, anche il poeta italiano Francesco Clodoveo Pentolini la celebra nei suoi Canti di donne illustri, (Livorno 1776: 199) dove compaiono anche le poetesse italiane medievali. "Ma non tacerà mai la Poesia/ la bellezza, l'ingegno, ed il valore/ della Contessa gloriosa Dia,/ cui spinse a morte un fiero aspro dolore./ Amò Guglielmo, ma l'iniqua e ria/ Sorte giunger lo fece all'ultim'ore./Perciò mai più non vide ora serena,/ e la vita lascio d'affanno piena/".
} 
ora comprendo di essermi sbagliata perché non gli ho donato il mio amore per cui ho vissuto nell'errore sia nel letto sia vestita. Come vorrei una sera tenere il mio cavaliere nudo tra le braccia ch'egli si considerasse felice se solo gli facessi da guanciale che mi lascia più incantata di quanto Fiorio di Biancofiore io gli dono il mio cuore e il mio amore la mia ragione, i miei occhi e la mia vita. Bell'amico amabile e buono quando vi terrò in mio potere potessi giacere al vostro fianco una sera e potessi darvi un bacio appassionato: Sapete di cosa avrei gran desiderio di avervi al posto del marito purchè mi concediate in pegno di fare ciò che voglio. (Martinengo 1996: 52)

Da un punto di vista linguistico e contenutistico è interessante notare come la costruzione utilizzata dalla Contessa "sono stata in grande errore ("don ai estat en gran error") ritorni nel sonetto di Compiuta Donzella "Alla stagion che il mondo foglia e fiora" 21 dove la poetessa sembra riprende questa costruzione quando accusa il padre di volerla dare in sposa ad un uomo che non ama ("Chè lo mio padre m'ha messa in errore,/ e tienemi sovente in forte doglia:/donar mi vole, a mia forza, signore).

Il tema del dolore, dell'affanno e dell'errore che Compiuta Donzella affronta nel suo testo trova un antecedente anche nella canzone di Clara D’Anduza, "En greu esmai et en greu pessamen" che inizia, infatti, con questi versi: "In grande angustia e grande tormento/han messo il mio cuore e in grande errore/". Una costruzione similare, sempre per quanto riguarda l'incipit è quella della composizione anonima intitolata "Amics en gran cossirier" (“Amico in grave tormento e in grande pena/ mi trovo per causa vostra/ e

\footnotetext{
${ }^{21}$ /Alla stagion che il mondo foglia e fiora,/accresce gioia a tutti i fini amanti:/ vanno insïeme alli giardini/ allora che gli augelletti fanno nuovi canti:/ la franca gente tutta s'innamora,/ ed in servir ciascun traggesi avanti,/ ed ogni damigella in gioi' dimora,/ a me n'abbondan smarrimenti e pianti./Chè lo mio padre m'ha messa in errore,/ e tienemi sovente in forte doglia:/ donar mi vole, a mia forza, signore./ Ed io di ciò non ho disio né voglia,/ e in gran tormento vivo a tutte l'ore:/ però non mi rallegra fior né foglia./
} 
del male che soffro/ non credo vi rendiate conto"). Le poetesse, in tutti e tre i casi, denunciano le difficoltà di poter essere libere e autonome nelle loro scelte e comportamenti, poiché la società patriarcale in cui vivono le opprime ed aspira a controllarle, esigendole determinati comportamenti.

L'amore non corrisposto è invece il tema di “A chantar m'er de so quieu non volria". Anche in questo testo, la Contessa de Dia utilizza un linguaggio diretto e traccia un bilancio della propria relazione amorosa, mettendo l'amante di fronte a tutte le sue manchevolezze, domandandogli le ragioni di un comportamento tanto crudele nei suoi confronti. Si tratta di una canzone in cui si possono rintracciare affinità e punti di contatto con il già citato sonetto di Monna Nina "Tapina me che amava uno sparviero".

A proposito di questo testo, già Paola Malpezzi Price (1988), segnalava i molti punti di contatto, anche da un punto di vista lessicale, con la poesia delle trobairitz. In particolare, osservava come la poetessa Alamanda nella sua tenzone con Guiraut de Bonelh, avesse già utilizzato il termine "mainieira" con l'accezione di docile, come lo userà anche Monna Nina (“a lo richiamo ben m'era maniero"). La poetessa italiana a differenza di Alamanda che con questa parola sottolineava come la fanciulla di cui parlava Guiraut non fosse affatto "docile", se ne serve per definire lo stato del suo amante/sparviero che si lascia governare/guidare. La descrizione di un amore in modo consapevole e razionale, costituisce una novità importante che ribalta la prospettiva tradizionale della lirica maschile e relega l'uomo, a oggetto descritto.

Un'altra composizione particolarmente significativa perché mostra un esempio di dialogo e complicità femminile è lo scambio poetico tra Almucs ed Iseo De Capio. Nella razó si racconta come Iseo De Capio preghi Almucs de Castelnau di perdonare Gigo de Tornen $^{22}$, colpevole di un grave torto nei confronti di Almucs. Non è l'unico caso di corrispondenza tra poetesse per quanto riguarda la lirica trovadorica, si pensi, ad esempio, alla tenzone a tre voci tra Alais, Iselda e Carenza, intitolata " $\mathrm{Na}$ Carenza al bel cors avinen", che costituisce un antecedente importante per quanto riguarda le poetesse italiane, in particolare per le petrarchiste marchigiane che, sempre attraverso la poesia,

\footnotetext{
${ }^{22}$ Gigo de Tornen potrebbe identificarsi con Guigo de Tournel e sarebbe stato l'amante di Almucs.
} 
creano un dialogo e un gioco poetico a distanza, si pensi al componimento che Elisabetta Trebbiani dirige a Livia Di Chiavello intitolato Trunto mio che le falde avvien che bacie ${ }^{23}$, dove il legame tra le due poetesse è rappresentato metaforicamente dall'immaginario incontro dei fiumi delle loro città, il Giano e il Tronto. Queste sentimento di sorellanza è presente anche nel testo "Coprite o muse" 24 che Leonora della Genga dedica ad Ortensia da Guglielmo in occasione della sua morte.

Di Azalais de Porcairagues, del distretto di Montpellier, si conserva un unico componimento "Ar em al freg temps vengut". In questo testo vi sono alcuni riferimenti biografici importanti, come quello alla possibile morte di Raimbaut d'Orange, avvenuta nel 1173, e il saluto finale rivolto ad Ermengarda di Narbona, mecenate trobadorica che protesse ed aiutò anche Azalais.

Nelle prime due stanze della canzone, l'autrice utilizza la descrizione del paesaggio per introdurre il tema doloroso che si andrà a trattare e per esprimere il suo stato d'animo. L'amore perduto l'ha svuotata lasciandola senza parole. La natura muta diventa lo specchio della sua anima e, attraverso le parole, riesce a sublimare il dolore e, quindi, a sopravvivergli:
Il tempo freddo è arrivato
con neve e ghiaccio e fango
e l'uccellino resta muto:
nessuno ha il coraggio di cantare

\footnotetext{
${ }^{23}$ Trunto mio, che le falde avvien che bacie/A la città de Pico, e più de Marte,/S'in mar, dove ogni fiume amistà facie,/T'incontrassi col Ian, diglie en disparte,/Ch'annunzi en nome mio salute, e pacie/a la mia Livia perita d'onn'arte:/La qual si à l'orecchi, ed occhi piacie,/o se veggia en persona, o scriva en carte./La carta bianca di più tu gl'accenna/Che del suo bel Paese ella me mandi/Per scrivervi sue gesta inclite, e sole./Ma più, che la sua carta, la sua penna/Vorrei, mentr'a laudar soi merti grandi/Sol la sua penna eloquente ce vole.

${ }^{24}$ Coprite o Muse di color funebre /Tutto Parnaso, e ogni luogo appesso,/Suelto il Lauro, piantate ivi il Cipresso./Sien le vostre querele ogn'hor più crebre./Il pianto, ch'uscirà da le palpebre/Empia Aganippe, e non si trovi in esso/Altro liquor, che quel che vi sia messo/Da gli occhi vostri, e da altrui tenebre./E poi c'harete con dolenti segni/Mostrati i danni sempiterni vostri/Per Ortensia gentile a tondo, a tondo./Direte a tutti i pellegrini ingegni;/Che spendano in lodare i sacri inchiostri/Questo spirto gentil si raro al mondo./ Il testo si trova citato ad esempio in Bergalli Gozzi, Luisa. 1726. Componimenti poetici delle più illustri rimatrici d'ogni secolo. Venezia: Antonio Mora. pt. 1: 5.
} 


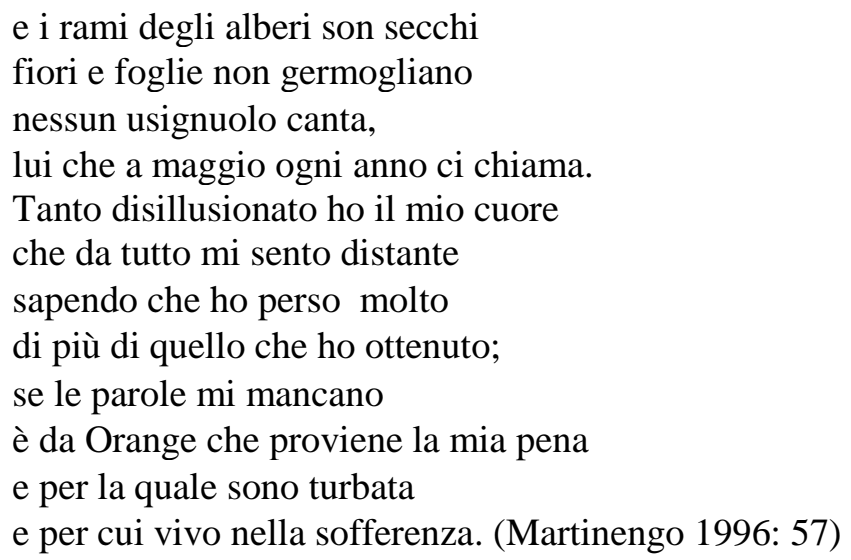

Un preludio che rimanda alla tradizione classica ma, che si ritrova anche nel testo di Compiuta Donzella "A la stagion che il mondo foglia e fiora", dove la poetessa si serve della descrizione della natura in fiore, con gli uccelli che cantano, per creare un forte contrasto con il suo stato d'animo di dolore. Anche nel caso di Azalais, ritorna il tema della corrispondenza tra donne, dal momento che il testo si dirige ad Ermengarda, donna mecenate che aiuta e protegge altre poetesse ed, ancora una volta, emerge il tentativo di costruzione di una linea genealogica al femminile, tra donne che scrivono e donne che le sostengono. Un altro partimen che può, in un certo senso, anticipare altre tematiche relative alle Querelle des femmes, è quello di Maria di Ventadorn, figura molto importante, anche per la sua attività culturale presso la corte di Ventadorn, una delle più note del tempo. Nel suo testo "Gui d'Uissel, be-m pesa de vos", la poetessa tratta con il poeta Gui d'Uissel delle differenze che intercorrono nelle relazioni amorose tra uomini e donne e insiste sulla reciprocità di diritti e doveri.

Anche un'altra poetessa, Castelloza, che visse probabilmente in Alvernia, nella sua canzone "Amics, s'ie.us trobes avinen" non esita a condannare le ipocrisie e gli inganni maschili, ("vi trovo malvagio, falso e vile") e nei suoi versi, abbandona l'idealizzazione dell'amore, scegliendo un linguaggio che rompa con le convenzioni e sfidi le norme sociali ("io so bene che a me piace così,/mentre tutti dicono sia sconveniente/ che una dama corteggi un cavaliere/"). Sempre Castelloza in "Mout avetz faich long estatge" diventa ancora più esplicita, dichiarando all'amante che desidera fare l'amore con lui ("vi 
chiedo di venire da me,/ dopo aver ascoltato/ questa canzone che vi garantisce/ che qui troverete una buona accoglienza") (Martinengo 1995: 84).

Infine, "Na Maria, pretz e fina valors" di Bieiris De Romans costituisce un caso abbastanza peculiare perché ha come destinataria un'altra donna e non fa in nessun momento riferimento ad un interlocutore maschile. Bieiris De Romans celebra l'amica Maria e la elegge a modello di donna ideale, capace di racchiudere in sè tutte le virtù, i meriti e gli onori, come accadrà per i già citati testi che Elisabetta Trebbiani e Leonora Della Genga indirizzano, rispettivamente a Livia Del Chiavello e Ortensia Da Guglielmo.

A distanza di secoli, i versi delle poetesse dell'al-Andalus e delle trobairitz colpiscono ancora per la loro immediatezza e per la capacità di superare i limiti dell'espressione femminile ed affrontare gli uomini sul terreno letterario, senza risparmiare critiche e senza il timore di uscirne sconfitte dialetticamente.

L'esperienza di queste autrici può allora essere letta ed interpretata come una pratica attiva, che rompe con la condizione di estraneità alla cultura da parte delle donne. Le poetesse italiane del Duecento e del Trecento ereditano formule e soggetti delle poetesse che le hanno precedute e, attraverso nuovi versi, continuano la Querelle des Femmes.

\section{Riferimenti bibliografici}

Aguadé Benet, Rosa Ma . 2011. "Cristine de Pizan y las trobairitz". La Querella de las mujeres siglos XIV-XV. Antecedentes de la polémica feminista. Ed. Cristina Seguro Graiño. Madrid: Asociación Al-Mudaya. 23-44.

Arriaga Flórez, Mercedes. 2008. "Le scrittrici marchigiane: un giallo letterario". Studi Umanistici Piceni. Sassoferrato: Istituto Internazionale di Studi Piceni, $n^{\circ}$ XXVIII: 161-168.

Arriaga Flórez, Mercedes, Daniele Cerrato, María Rosal Nadales. 2012. Poetas italianas de los siglos XIII y XIV en la Querella de las mujeres. Sevilla: Arcibel.

Bergalli Gozzi, Luisa. 1726. Componimenti poetici delle più illustri rimatrici d'ogni secolo. Venezia: Antonio Mora. 
Bogin, Magda. 1976. The women trobadours. New York: Paddington Press.

Cabanilles Sanchís, Antonia. 1992. "El discurso de las trobairitz: un modelo de enunciación dialógica". Actas del IX Simposio de la Sociedad Española de $l$ Literatura General y Comparada. Zaragoza: Universidad de Zaragoza, tomo I: 7584.

Cerrato, Daniele. 2013. "Filoginia e querelle de femmes tra Duecento e Quattrocento in Italia". Las relaciones ítalo-españolas: traducción, lengua y literatura. Eds. Estela González de Sande, Mercedes González de Sande. Sevilla: Arcibel. 149-166.

Gangutia, Elvira. 1972. Poesía griega de amigo y poesía arábigo-española. Emerita, 40: 329-396.

Garulo, Teresa. 1989. "Sobre la poetisas en al Andalus". La mujer en al-Andalus. Reflejos históricos de su actividad y categorías sociales. Ed. María Jesús Viguera. Madrid: Universidad Autonoma de Madrid. 191-199. 1986. ed. Diwan de las poetisas de al-Andalus. Madrid: Hiperión.

González de Sande, Estela. et. al. 2013. Poetas cortesanas en la querella de las mujeres. Sevilla: Arcibel.

Malpezzi Price, Paola. 1988. “Uncovering Women's Writings: Two Early Italian Women Poets". Journal of the Rocky Mountain Medieval and Renaissance Association, 9: $1-15$.

Martinengo, Marirí. 1996. Le trovatore: poetesse dell'amor cortese (testi provenzali con traduzione a fronte). Milano: Libreria delle donne di Milano.

Massagrande, Matteo. 1995. Lamento della sposa padovana: quattro testi poetici e quattro incisioni. Belluno: Proposte d'arte Colophon.

Millet, Víctor. 2006. "Los cancioneros de la poesía cortesana alemana anterior a 1350: características y problemas". Convivio, estudios sobre la poesía de cancioneros. Granada: Universidad de Granada.

Muñoz Saavedra, María Paz, Juan Carlos Búa Carballo. 2007. Lírica medieval alemana con voz femenina (siglos XII-XIII). Valladolid: Universidad de Valladolid.

Paden, William D. 1989. The voice of the Trobairitz. Perspectives on the Women Troubadours. Filadelfia: University of Pennsylvania Press.

Ramírez Almazán Dolores, Milagro Martín Clavijo, Juan Aguilar González, Daniele Cerrato (eds.). 2011. La Querella de las mujeres en Europa e Hispanoamérica. Sevilla: Arcibel.

Rieger, Angela. 1991. Trobairitz. Der Beitrag der Frau in der altokzitanischen höfischen Lyrik. Edition des Gesamtkorpus. Tübingen: Max Niemeyer Verlag. 
Rubiera Mata, María Jesús (ed.). 1990. Poesía femenina hispanoárabe. Madrid: Editorial Castalia.

Viguera, María Jesús. 1989. La mujer en al-Andalus. Reflejos históricos de su actividad y categorías sociales. Madrid: Universidad Autonoma de Madrid.

Víñez Sánchez, Antonia. 2013. "La voz disidente de las trobairitz". El eterno presente de la literatura. Estudios literarios de la Edad Media al siglo XIX. Eds. María Teresa Navarrete Navarrete, Miguel Soler Gallo. Roma: Aracne. 35- 63. 